\title{
Permanent neurological deficits complicating sinoatrial block
}

\author{
H. H. R. Samarasinghe and Nimal Senanayake \\ From the Department of Medicine, University of Ceylon, Colombo, Ceylon
}

Two cases are reported of sinoatrial block complicated by recurrent neurological manifestations leading to permanent disability. In one case where a demand pacemaker was introduced there was complete abolition of the recurrent symptoms. Neurological manifestations were probably the result of cerebral embolism due to accompanying paroxysms of atrial fibrillation or cerebral thrombosis.

Adams-Stokes attacks and other cerebral dysfunctions secondary to atrioventricular (AV) block are well known. But the possibility of such manifestations due to disease or malfunction of the sinoatrial (SA) node was not appreciated until Short (1954) described a syndrome of alternating bradycardia and supraventricular tachycardia associated with syncope in 4 patients. These patients were noted to have Received 2 October 1972. slow sinus rates with periods of 'sinus standstill' in two. Birchfield, Menefee, and Bryant (1957) and Herbinger (I96I) subsequently described similar patients with bradycardia, asystole, paroxysmal atrial fibrillation, and syncope. Reviewing these reports and their own cases, Easley and Goldstein (197I) highlighted a syndrome characterized by syncope due to sinoatrial block and sinus bradycardia, in addition to a complete spectrum of supra-

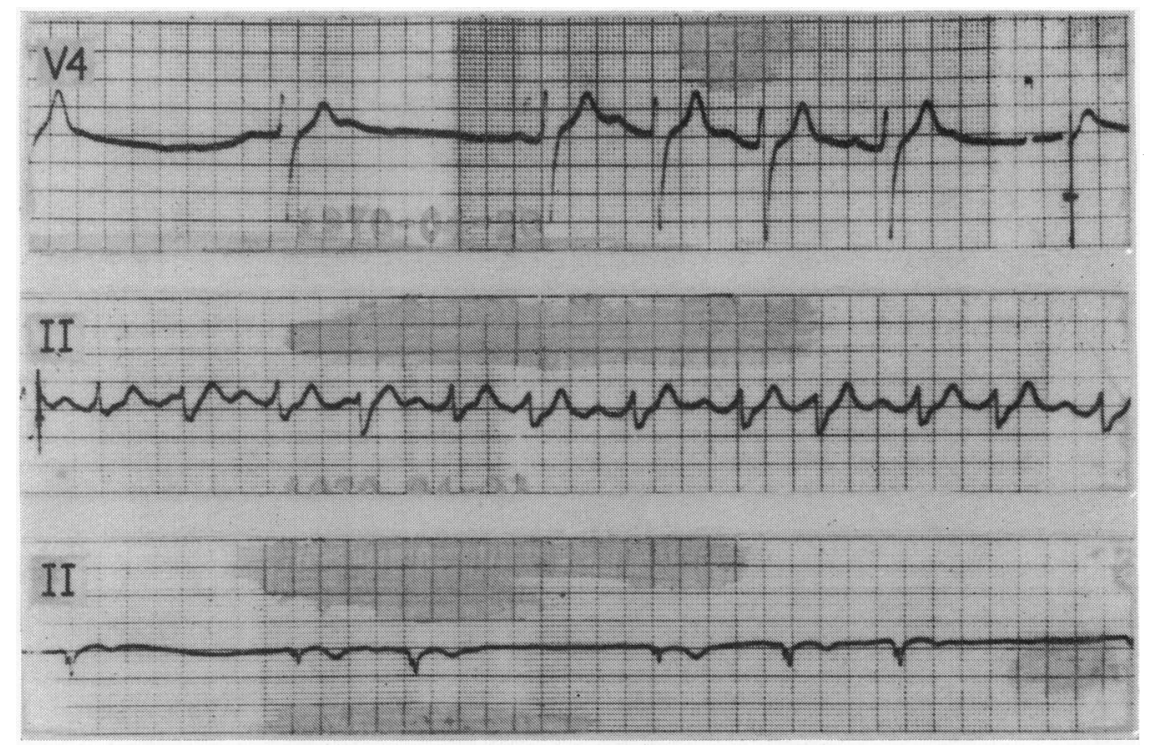

FIG. I Serial electrocardiograms of Case I showing sinoatrial block with escape atrioventricular junctional rhythm and fast atrial fibrillation in the second rhythm strip. 
ventricular tachycardias which they termed 'sinoatrial syncope'.

The purpose of this paper is to document two cases of the above syndrome who developed a hitherto unreported complication of sinoatrial block, namely cerebrovascular accidents causing permanent neurological deficits.

\section{Case reports}

\section{Case I}

A man aged 4I years was first investigated at the University Medical Unit, Colombo, in January 1969 for recurrent attacks of dizziness. He was noted to have bradycardia alternating with atrial flutter. He was normotensive. Nothing relevant could be found in his past history or in the family history to account for the arrhythmia. The patient left the ward on no specific treatment as his symptoms improved. A year later he developed numbness of the left half of his body and felt faint. These symptoms had come on quite abruptly. He then had a generalized convulsion and was unconscious for a few minutes. On regaining consciousness he noticed weakness of the left side of his body. He did not experience chest pain, sweating, or dyspnoea during this period. Two more convulsions occurred over the next two hours and the patient was admitted to hospital.

On admission the patient was afebrile. His pulse was 96/min, irregular with occasional dropped beats and was of moderate volume. The blood pressure was Iro/80
mmHg. The rest of the cardiovascular system and the respiratory system were clinically normal. The liver and spleen were not palpable. On examination of the central nervous system the patient was conscious and rational. He was right-handed. He had dysarthria with slurring of speech. There was no neck stiffness. The left pupil was small but reacted to light and accommodation. The right pupil was normal. There was slight ptosis on the left side. A horizontal nystagmus with the quick component to the left was detected. Other cranial nerves including the ocular fundi were clinically normal. The limbs were hypotonic and clumsy on the left side, with evidence of incoordination on the same side. Plantar reflexes were flexor bilaterally. All modalities of sensations were intact. The results of the investigations done at this stage were as follows: urine for sugar and albumin, nil; ESR (Ist hour) $5 \mathrm{~mm}$; WBC $8600 / \mathrm{mm}^{3}$, with a normal differential count; haemoglobin content $12.9 \mathrm{~g} / 100 \mathrm{ml}$; toxoplasma agglutination test and the serological tests for syphilis negative. Skull $x$-ray, carotid and vertebral angiograms, and the electroencephalogram were normal. Serial electrocardiograms are shown in Fig. I.

The patient was managed conservatively and he left the ward after two weeks. No specific treatment was given for the arrhythmia. The neurological deficits improved to a considerable degree. In October 1970 he had an attack of transient hemiparesis involving the right side of the body. In May I97I he was readmitted with aphasia and right-sided hemiplegia. His electrocardiogram persistently showed evidence of sinoatrial block

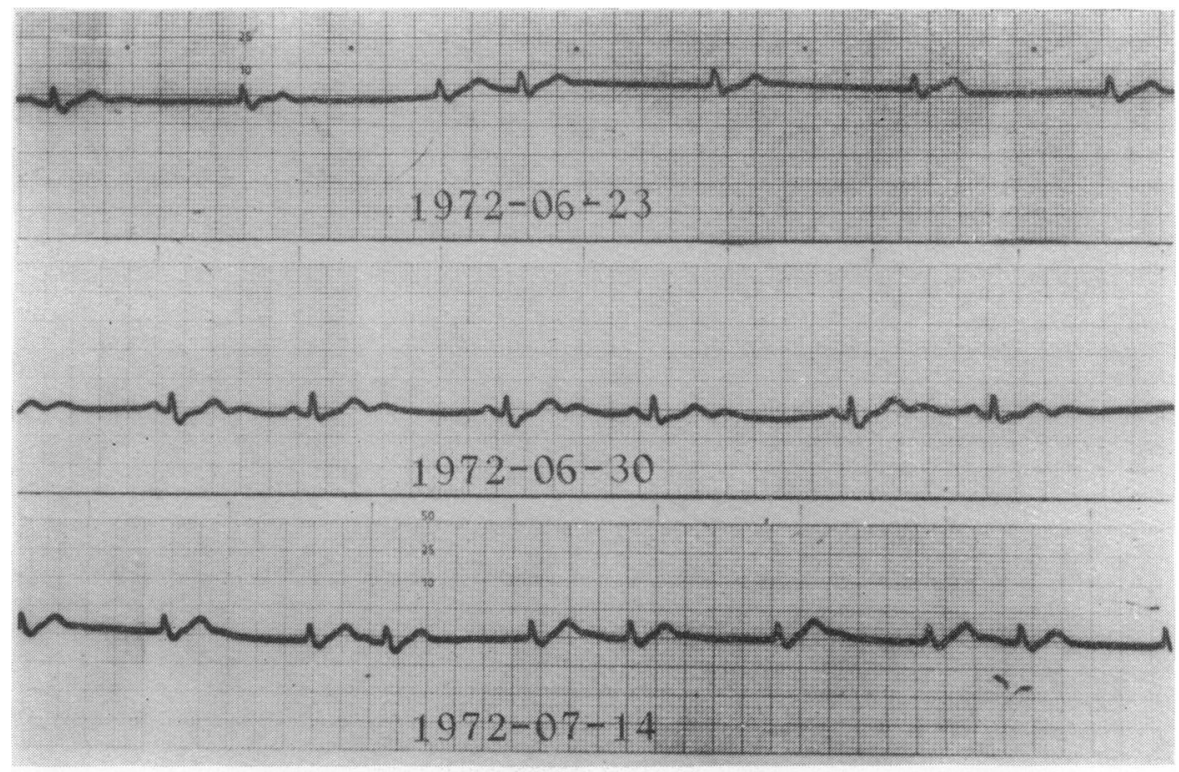

FI G. 2 Serial electrocardiograms of Case 2 showing sinoatrial block with escape atrioventricular junctional rhythm. 
with junctional escape rhythm. Considering the recurrent nature of his symptoms, in August 1971 a demand pacemaker was inserted to correct his cardiac arrhythmia. With this, the attacks of dizziness completely disappeared. Except for the residual disability of hemiplegia he had been quite asymptomatic up to the time of writing this paper.

\section{Case 2}

A 39-year-old man was transferred from a district hospital to the University Medical Unit, Colombo, in June 1972, with a history of paralysis of the right side of the body. Three days before admission he had suddenly felt dizzy and weak. Within a few minutes he was aphasic and developed paralysis of the right side of the body. He neither had fits nor lost consciousness. He did not have chest pain or sweating. At the district hospital he was treated symptomatically with no specific drugs. Three days later he came to us. It was then revealed that in December 197I he had had a similar attack of aphasia with hemiplegia which improved without any specific measures. He was not on any drugs and was in reasonably good health until he had the present episode. He had no history of hypertension, ischaemic heart disease, or rheumatic fever in the past. There was no family history of diabetes mellitus.

On examination the patient was afebrile. The pulse was $42 / \mathrm{min}$, irregular with dropped beats and was of moderate volume. The blood pressure was 100/50 $\mathrm{mmHg}$. The rest of the cardiovascular system and the respiratory system were clinically normal. The liver and spleen were not palpable. On examination of the central nervous system the patient was conscious but aphasic. $\mathrm{He}$ was right-handed. The pupils and the ocular fundi were normal. There were no signs of meningeal irritation. Results of the investigations carried out were as follows: urine for sugar and albumin, nil; ESR (Ist hour) $9 \mathrm{~mm}$; WBC $10,200 / \mathrm{mm}^{3}$, with a normal differential count; haemoglobin content $10.1 \mathrm{~g} / 100 \mathrm{ml}$; blood urea $30 \mathrm{mg} / 100 \mathrm{ml}$. Total serum lactic dehydrogenase 6I IU (normal 50-170 IU). Heat stable fraction 57 per cent of the total (normal 30-60\%); 2-hour postprandial blood sugar $70 \mathrm{mg} / 100 \mathrm{ml}$; serum cholesterol $232 \mathrm{mg}$ / $100 \mathrm{ml}$. Toxoplasma haemagglutination test and the serological tests for syphilis were negative; blood for arborviral antibodies did not show a rising titre during the first two weeks. CSF was clear, with no abnormality in the constituents. Left percutaneous carotid angiogram and the electroencephalogram were normal. Serial electrocardiograms are shown in Fig. 2.

Treatment was begun with ephedrine and later changed over to long-acting isoprenaline - 'saventrine' as there was no improvement. With 'saventrine' the bradycardia improved but the sinoatrial block persisted with escape junctional rhythm. Aphasia and hemiplegia improved partially, leaving some residual neurological deficits.

\section{Discussion}

Sinoatrial block is a disturbance of cardiac rhythm in which the atrial response is delayed or omitted because of partial or complete interference with the propagation of impulses from the sinoatrial node to the atria. When this leads to the omission of two, three, or more successive atrial beats, 'atrial standstill' occurs. In these instances there is a tendency for the ventricles to be driven by a lower pacemaker which is usually located near the AV junction. Occasionally prolonged atrial standstill develops without the appearance of an AV nodal or other idioventricular pacemaker giving rise to an AdamsStokes attack (Friedberg, 1966). In the two cases that have been described all these phenomena were seen to a varying degree during the course of the illness. The unusual feature in both of them was the recurrent neurological manifestations that led to permanent residual disability. In Case I the initial episode was due to brain-stem ischaemia resulting in cerebellar signs, dysarthria, and a Horner's syndrome. He recovered from it and developed a cerebral infarct in the left hemisphere giving rise to hemiplegia and aphasia. Case 2 probably had a transient ischaemia followed by an infarct in the left cerebral hemisphere. These recurrent multiple ischaemic episodes seem to be the result of cerebral embolism. Systemic embolism from thrombi formed in the left atrium is a well-recognized complication of atrial fibrillation. It is also interesting to note that atrial fibrillation itself is a frequent accompaniment of sinoatrial block (Rokseth et al., 1970; Easley and Goldstein, 197I). In this study too, electrocardiographic evidence of atrial fibrillation has been recorded on one occasion in Case I. Though not detected clinically or electrocardiographically, the possibility of transient atrial fibrillation cannot be excluded in Case 2 as he has not been continuously monitored. Thus, it seems reasonable to conclude that the cause of the cerebral embolism in our patient probably was the result of paroxysmal atrial fibrillation that accompanied SA block.

Another possible explanation may be offered by considering recurrent cerebral thrombosis as the cause of the ischaemic attacks. Thrombosis may result from sudden lowering of blood pressure in the cerebral blood vessels during a period of prolonged atrial standstill with ventricular asystole as could occur in sinoatrial block, probably by a longer period of cardiac standstill than the one that would produce an Adams-Stokes attack. Cerebral thrombosis due to sudden lowering of blood pressure is a recognized complication of acute myocardial infarction (Glathe and Achor, 1958). However, our patients had no clinical, electrocardiographic, or biochemical evidence of acute myocardial infarction.

Artificial pacing as the treatment of choice in sinoatrial syncope was first described by Muller and Finklestein in 1966. Abolition of the recurrent 
symptoms after correction of the arrhythmia by means of the pacemaker in Case I supports the view that the previous neurological manifestations were the result of sinoatrial block. It also goes to show that the early recognition of sinoatrial block and its proper treatment can prevent the occurrence not only of syncopal attacks, cardiac failure, and a spectrum of tachyarrhythmias which may occasionally be fatal, but also of a variety of neurological manifestations that may lead to permanent disability.

We wish to thank Dr. R. Natkunam for inserting the pacemaker in Case I and Dr. N. J. Wallooppillai for advice on the preparation of this paper.

\section{References}

Birchfield, R. I., Menefee, E. E., and Bryant, G. D. N. (1957). Disease of the sinoatrial node associated with bradycardia, asystole, syncope, and paroxysmal atrial fibrillation. Circulation, 16, 20.
Easley, R. M., and Goldstein, S. (I97I). Sino-atrial syncope. American fournal of Medicine, 50, 166.

Friedberg, C. K. (1966). Diseases of the Heart. Saunders, Philadelphia and London.

Glathe, J. P., and Achor, R. W. P. (1958). Frequency of cardiac disease in patients with strokes. Proceedings of the Staff Meetings of the Mayo Clinic, 33, 4I7.

Herbinger, W. (196I). Increasingly frequent syncopes caused by sino-auricular block. Cardiologia, 38, 267.

Muller, O. F., and Finklestein, D. (1966). Adams-Stokes syndrome due to sinoatrial block. American fournal of Cardiology, 17, 433.

Rokseth, R., Hatle, L., Gedde-Dahl, D., and Foss, P. O. (1970). Pacemaker therapy in sino-atrial block complicated by paroxysmal tachycardia. British Heart fournal, 32, 93.

Short, D. S. (1954). The syndrome of alternating bradycardia and tachycardia. British Heart fournal, 16, 208.

Requests for reprints to Dr. H. H. R. Samarasinghe, Department of Medicine, Faculty of Medicine, University of Ceylon, Kynsey Road, Colombo 8, Ceylon. 